ectopic pregnancy overall the number of women hospitalised decreased from 1204 to 1192, a decrease in annual rates from 14.3 [13.5 to 15.1] to 12.6 [11.9 to 13.3] per 1000 births ( $p$ trend $<0.001$ ) but this decrease was mostly in women aged between 25 to 44 years. There was no appreciable fall in hospitalisations for ectopic pregnancy in women aged 15 to 24 years (13.1 [11.5 to 15.0 ] to 13.1 [11.4 to 15.0] per 1000 births; ptrend=0.9; see Abstract P1-S3.01 figure $1 \mathrm{~B})$.

Conclusions Among women aged 25 to 44 years decreases in hospitalisations for ectopic pregnancy and increases in hospitalisations for infertility were observed. The relationships between chlamydia prevalence, testing and notifications do not appear to be straightforward.

\section{P1-S3.02 THE ECONOMIC BURDEN OF CHLAMYDIA AND GONORRHOEA IN CANADA}

doi:10.1136/sextrans-2011-050108.135

L Smylie, P Lau, R Lerch, C Kennedy, R Bennett, B Clarke, A Diener. Public Health Agency of Canada, Ottawa, Canada

Background The latest estimate of the economic burden of chlamydia and gonorrhoea in Canada was modelled with data from 1990 and was published in 1992 by Ron Goeree and Paul Gully. Given the changes that have occurred since in diagnostic testing technology, the availability of new data sources and increasing rates of the two infections, a new model using updated data from 2000 is called for.

Methods Diagnostic test costs were estimated using provincial laboratory data on the number of diagnostic tests performed for Chlamydia trachomatis (CT) and Neisseria gonorrhoeae (GC) for the year 2000. Direct costs of CT and GC from drugs, hospitals, and physician billings were estimated using the Economic Burden of Illness in Canada (EBIC) data. Direct costs of associated sequelae of each infectious disease were also included in the model. Indirect costs estimated in the model included production losses from both infectious diseases and their associated sequelae. Sensitivity analyses were conducted to provide upper, base and lower-bound estimates of the total cost.

Results The preliminary combined estimate for both direct and indirect costs of CT and GC (in 2000 dollars) ranges from approximately $\$ 31.5$ to $\$ 178.4$ million (CAD).

Conclusions Further work is required to improve data access and estimates of the burden of infection in Canada. A top priority should be to improve the data infrastructure by expanding data linkages within and among provinces around laboratory tests. The majority of costs related to CT and GC are attributable to drug, hospital and physician costs, suggesting that much of the burden of these two infections can be reduced through implementation of effective prevention programs. The number of CT infections has increased exponentially since 2000, warranting further modelling considering current incidence rates and inflation costs.

\section{P1-S3.03 MORTALITY AMONG MEN WHO HAVE SEX WITH MEN SEEN IN STD CLINIC PATIENTS: HIGH RATES OF SUICIDE AND LOW RATES OF ANAL CANCER DEATH}

doi:10.1136/sextrans-2011-050108.136

${ }^{1} \mathrm{~S}$ Glick, ${ }^{2} \mathrm{R}$ Kerani, ${ }^{2} \mathrm{M}$ Golden. ${ }^{1}$ University of Washington, Seattle, USA; ${ }^{2}$ Public Health, Seattle and King County, Seattle, USA

Background Because men who have sex with men (MSM) are not identifiable at a population level, there are few data on cause-specific mortality rates among MSM. MSM are more likely to report suicide attempts than heterosexuals, but it is not known if suicide mortality is higher among MSM. Likewise, although anal cancer is more common among MSM than among heterosexuals, the absolute risk of anal cancer death among MSM is not known. We compared suicide and anal cancer mortality rates among MSM and heterosexual males and females in an STD clinic population.

Methods We enumerated all Public Health-Seattle and King County STD Clinic attendees from 1993-2007 $(n=86,807)$. Using 1993-2008 mortality data from Washington State $(n=702850)$, we identified STD clinic attendees who had died and their underlying cause of death. We calculated suicide and anal cancer mortality rates for MSM, heterosexual men, and women, and standardised these rates using the age, race, and gender distribution of King County residents (US Census, 2000). We calculated adjusted incidence rate ratios (IRR) among STD clinic attendees using Poisson regression. To compare mortality rates among STD clinic attendees to the general population, we calculated rates in King County.

Results Among STD clinic attendees, 14\% were MSM, 52\% were heterosexual men, and 34\% were women. For the period between 1993 and 2008, we matched 1901 Washington state deaths to STD clinic attendees for an overall unadjusted mortality rate of 1.8 deaths per 1000 person-years. The standardised suicide mortality rate among MSM was 0.20 per 1000 person-years, compared with 0.11 among heterosexual men and 0.09 among all women. Adjusted for age and race, among STD clinic attendees, MSM were 1.6 times more likely to die from suicide than heterosexual men (IRRadj=1.6, $95 \%$ CI 1.02 to 2.61 ) and nearly four times more likely than women (IRRadj=3.9, 95\% CI 2.14 to 7.24 . The suicide mortality rate among MSM in the STD clinic was higher than the rate among all men in King County (standardised rate ratio $=1.5$ ), adjusted for age and race. We identified no deaths due to anal cancer in any subpopulation (95\% CI 0 to 0.4 per 100000 person-years).

Conclusions These data suggest that, at least among STD clinic attendees, MSM are approximately twice as likely to die from suicide as heterosexual men, and these rates are higher than the general population. Anal cancer death rates among King County MSM appear to be low.

\section{P1-S3.04 DEATH IN THE ERA OF HAART: NOT ALL ABOUT AIDS}

doi:10.1136/sextrans-2011-050108.137

R Smith, M Kall, V Delpech. Health Protection Agency, London, UK

Background Mortality from AIDS has dramatically reduced since the introduction of effective antiretroviral therapy, Nevertheless, HIV infected individuals remain at higher risk of early death. We investigate all causes of deaths among HIV diagnosed adults reported nationally in the HAART era.

Methods Analyses of data from national HIV surveillance linked to the Office National Statistics. Deaths among all adults $(\geq 15)$ diagnosed between 1997 and 2008 in England and Wales (E\&W) were reviewed and grouped by cause of death (COD) and risk information. Death rates were calculated using a census of all persons accessing HIV care in 2008 (SOPHID).

Results The crude mortality rate among HIV-infected individuals (15-59yrs) fell from 21.8 to 8.2 per 1000 persons living with diagnosed HIV in 2008. A total of 3640 (5.6\%) persons diagnosed over the 12-year period were reported to have died [3544 had a cause of death]. The overall median age at death was 41 yearss [IOR: $35-50$ years]. AIDS related mortality accounted for $56 \%$ of all deaths: $51 \%$ among MSM, 61\% among heterosexuals and $34 \%$ among IDUs. AIDS-related respiratory infection (including PCP) was the most common COD in MSM and heterosexuals whereas 
external causes were the most common COD among IDUs. Non AIDS mortality increased sixfold since 1997 (36-226), with particular increases in cardio/cerebrovascular disease, non-AIDS defining malignancies and external causes. Infectious diseases (mostly respiratory) was the most common underlying cause of non AIDS deaths for MSM and heterosexuals aged $15-59$ : (38\% and $36 \%$ respectively) and also high among IDUs (35\%). External causes (mostly due to overdose and suicide) was the most common COD among IDUs (36\%) and high among MSM (19\%). Cardio/cerebrovascular disease and non AIDS malignancies accounted for $16 \%$ and $11 \%$ of nonAIDS deaths (15-59 years) respectively. Three quarters $(76 \%)$ of all AIDS deaths and $47 \%$ of non AIDS deaths occurred within a year of diagnosis.

Conclusion Overall mortality rates in HIV positive persons have substantially declined in the HAART era but remain high compared to the general population. Clinical AIDS associated with late presentation continues to account for the majority of deaths. Our analyses also reveal that a disproportionate number of deaths are due to infectious diseases, overdose and suicide occur in this population, many of which may be preventable. Surveillance of nonAIDS causes of death is critical in the HAART era.

\section{P1-S3.05 ATTRIBUTABLE PROPORTION OF TUBAL FACTOR INFERTILITY CAUSED BY CHLAMYDIA: AN ESTIMATE BASED ON SEROLOGICAL EVIDENCE ADJUSTED FOR TEST RESOLUTION}

doi:10.1136/sextrans-2011-050108.138

M Price, A E Ades, J Macleod, P Horner. University of Bristol, Bristol, UK

Background Many published studies compare the prevalence of Chlamydia trachomatis (CT) antibody in women with Tubal Factor Infertility (TFI) and a control group. In principle these studies can be used to estimate the attributable fraction of TFI caused by CT, however it is necessary to account for the sensitivity and specificity of the antibody tests employed.

Methods We use sensitivity and specificity estimates from a discrepancy analysis by Morre (2002), and a study by Wills (2009) in which specificity of antibody test results was assessed in children, to derive estimates of the resolving power of three peptide-based assays and MIF; this is a reflection of the difference between sensitivity and false positive rates. Based on studies of antibody levels in different settings we adopt a model which assumes that antibody levels in women whose TFI is caused by CT are higher than levels in control women or those whose TFI has other causes. Applying this model to the data from Land (2003) we find strong support for the hypothesis of higher antibody levels in CT-related TFI, and for the estimates of test resolution from earlier studies. Using a range of assumptions about cumulative incidence of CT in the control group we were able to derive a range of estimates for the proportion of TFI cases caused by CT.

Results Our results suggest that the sensitivity of antibody tests in women whose TFI was caused by CT is higher than in women who have previously had CT but whose TFI was due to another cause and control women. Based on our estimates of the resolving power of the tests from the Morre (2002) and Wills (2009) studies we estimate the proportion of TFI episodes that are due to Chlamydia to be between $20 \%$ and $45 \%$. Conclusions By adjusting for the sensitivity and specificity of tests it is possible to derive a quantitative estimate of the causal rate of CT in TFI. Taken together with other findings our results suggests that detailed studies of antibody levels can be used to shed further light on the causal rate of CT.

\section{Epidemiology poster session 3: Burden of disease: PID \\ P1-S3.06 PREVALENCE AND DETERMINANTS OF NEISERIA GONORHOEA AND CHLAMYDIA INFECTIONS AMONG GYNAECOLOGICAL PATIENTS WITH PELVIC INFLAMMATORY DISEASE AT UNIVERSITY TEACHING HOSPITAL, LUSAKA}

doi:10.1136/sextrans-2011-050108.139

P Yassa. University Teaching Hospital, Lusaka, Zambia

Methodology This was a descriptive cross sectional study conducted on women presenting clinically with pelvic inflammatory disease to the outpatient department of obstetrics and gynaecology at University Teaching Hospital in Lusaka. Behavioural and demographic variable were collected through questionnaire. Endo-cervical smear was obtained and screened for gonorrhoea and Chlamydia using respective rapid test and gram stain for gonorrhoea.

Results 43 (37\%) of a total 116 respondents had gonorrhoea but no Chlamydia was isolated. 114 (98.3) had sexual partners. 101 had steady sexual partners, 19 had casual partners and 9 had anonymous sexual partners with 37 (36.6\%), 10 (52.6\%) and 6 (66.7\%) gonorrhoea isolation respectively. Some had multiple sexual partners. Gonorrhoea was isolated from 4 (28.6\%) of the 14 respondents who had one new sexual partner, and all who had two or more sexual partners had gonorrhoea isolated. Gonorrhoea isolation in relation to frequency of sexual intercourse per week was as follows: once 1/13 $(7.7 \%)$, twice $2 / 11(18.2 \%)$, thrice $3 / 11$ (27.3\%) and more than three times 32/65 (49.2). Gonorrhoea was also higher in those who had sex with casual or anonymous sexual partner under influence of alcohol $6 / 11(54.5 \%)$ or obtained anonymous sexual partner from Market, shopping canter $4 / 5(80 \%)$, street, bar, disco, or night club 7/9 (77.8\%). Gonorrhoea detection was as follows: 18/36 (50.0\%) for those with adnexial tenderness, 21/45 (46.7\%) with inflamed cervix, 37/92 (40.2\%) with lower abdominal tenderness and 32/87 (36.8\%) with normally appearing cervix.

Conclusion The prevalence of Neiseria gonorrhoea was 37\% detected. There was no Chlamydia isolated. Low socio-economic status and young age were the higher risk. The sexual risk behaviours associated were; the number of casual or anonymous sexual partners, and non-use of condoms. Lower abdominal pain and tenderness with cervical motion and adnexial tenderness were the major sign.

\section{P1-S3.07 RELATION BETWEEN FEMALE INFERTILITY AND SEXUALLY TRANSMITTED GENITAL INFECTIONS}

doi:10.1136/sextrans-2011-050108.140

C Almanza, M Ricardo, A González. Cira Garcia Clinic, Havana, Cuba

Background Sexually transmitted genital infections can bring about severe consequences, among them the leading cause of pelvic inflammatory desease (PID), which can lead to infertility. The objective of this study was to determine the relation between female infertility and sexually transmitted genital infections.

Methods An analytic study of cases and controls was carried out in Ramón González Coro Gyneco-obstetric Hospital, Cuba, 2009. The studied cases were 89 infertile women with tube obstruction who were assessed in infertility consultations, and the controls were carried out in 100 pregnant women who were about to give birth. Vaginal and endocervical secretion samples were taken. Genital micoplasma was present; it was determined through bacteriological culture techniques. Trichomonas vaginalis, Candida spp, Bacterial vaginosis, Neisseria gonorrhoeae, Chlamydia trachomatis, as well as the presence of other genital pathologicals; were investigated. The test 